\title{
Formulation and Evaluation of Ibuprofen Loaded Lipospheres for Effective Oral Drug Delivery
}

\author{
M.A. Momoh ${ }^{1}$, F.C Kenechukwu², M.S. Gwarzo ${ }^{3}$ and P.F. Builders \\ ${ }^{1}$ Drug Delivery Research Unit, Department of Pharmaceutics, University of Nigeria, Nsukka-410001, Nigeria \\ ${ }^{2}$ Department of Pharmaceutics and Pharmaceutical Microbiology, Usmanu Danfodiyo University, \\ Sokoto, Nigeria \\ ${ }^{3}$ Department of Pharmaceutical Technology and Raw Material Development, National Institute for \\ Pharmaceutical Research and Development, Abuja, Nigeria
}

Received: April 05, 2014; Accepted: December 10, 2014; Published (web): February 09, 2015

\begin{abstract}
Ibuprofen (IBU) is an anti-inflammatory drug characterized by low solubility and bioavailabilty. This study was to develop IBU-liposphere and investigated for in vitro and in vivo performance. IBU free base was incorporated into lipospheres based on micronized beeswax and Phospholipon ${ }^{\circledR} 90 \mathrm{H}$ in the ratio of (1:3), via hot emulsification. IBU-loaded lipospheres were characterized based on morphology, encapsulation efficiency (EE\%), and in vitro drug release. Analgesic, anti-inflammatory activities and the pharmacokinetics were similarly evaluated. Minimum and maximum encapsulation efficiency (EE\%) of 89.4 and $97.9 \%$ were obtained for lipospheres A1 and A3, respectively. Stable, spherical and smooth lipospheres of size range $101 \pm 0.30$ to $178 \pm 0.30 \mu \mathrm{m}$ were obtained. Minimum and maximum release of 75 and $96.9 \%$ were obtained for A1 and A3, respectively. Significant $(\mathrm{p}<0.005)$ analgesic and anti-inflammatory activities were achieved with prolong plasma concentration. IBU-lipospheres based on beeswax and phospholipid could be explored as an alternative drug delivery system.
\end{abstract}

Key words: Ibuprofen, lipospheres, drug delivery, sustained-release, characterization

\section{INTRODUCTION}

The main role of lipid-based formulations has traditionally been to improve the solubility of sparingly water soluble drugs especially Biopharmaceutics Classification System (BCS) Classes II and IV drugs. ${ }^{1}$ However, the spectrum of application of lipid-based formulations has widened as the nature and type of active drugs under investigation vary. Lipid-based formulations may also protect active compounds from biological degradation or transformation, which in turn can lead to an enhancement of drug potency, high bioavailability, controlled drug release and low toxic effect. $^{2-4}$ The most frequently used lipid formulation is lipospheres due to it numerous advantages. ${ }^{5}$

Correspondence to: M.A. Momoh

Tel : +234 (0)8037784357

E-mail: jointmomoh@yahoo.com; audu.momoh@unn.edu.ng

Dhaka Univ. J. Pharm. Sci. 14(1): 17-27, 2015 (June)
Lipospheres are solid water-insoluble microparticles with solid hydrophobic core and a layer of phospholipids embedded on the surface of the core shielding a biologically active agent in the core. $^{3-5}$ The average particle diameter of liposphere is between $0.3-250 \mu \mathrm{m}$ based on an earlier researcher. ${ }^{6}$ Lipospheres have several advantages over other lipid delivery systems such as emulsions, vesicles, and liposomes, in terms of stability, low cost of reagents, ease of production, high dispersibility in aqueous medium, and a release rate that is controlled by the phospholipid coating and the carrier. Lipospheres also have a lower risk of drug reaction than with emulsion systems because the vehicle is inert solid material. ${ }^{7}$ Moreover, the release rate of a substance from the lipospheres can be manipulated by altering either or both inner solid vehicle or the outer phospholipids. ${ }^{6-8}$ In addition, it can be used to mask taste and odor in oral formulations. ${ }^{9}$ The lipid component of the lipospheres can significantly 
enhance epithelial permeability and increase the oral bioavailability of active pharmaceutical ingredients (APIs). ${ }^{3}$

Ibuprofen (IBU) is a well-established nonsteroidal anti-inflammatory drug (NSAID) used for a variety of pain and inflammatory conditions such as musculoskeletal disorders (e.g. rheumatoid arthritis and juvenile rheumatoid arthritis). ${ }^{10}$ IBU is classified by BCS as a Class II API as it presents low solubility and high permeability. ${ }^{11}$ Thus, increasing the solubility of IBU is the major obstacle to enhancing its bioavailability. ${ }^{12}$ Previous studies indicate that polymer based IBU microparticles are often characterized by a high initial burst effect resulting from the accumulation of drug crystals on the surface of the microparticles. ${ }^{13-15}$ In some cases, the formulated IBU microparticles were unsuitable for well controlled release. The methods in these formulations were cumbersome and scaling up very expensive. ${ }^{16}$

Homolipids are esters of fatty acids with alcohols. They are lipids containing only carbon (C), hydrogen $(\mathrm{H})$, and oxygen $(\mathrm{O})$, and as such are referred to as simple lipids. ${ }^{8}$ Heterolipids are lipids containing nitrogen $(\mathrm{N})$ and phosphorus $(\mathrm{P})$ atoms in addition to the usual $\mathrm{C}, \mathrm{H}$ and $\mathrm{O}$ e.g. phospholipids, glycolipids and sulfolipids. They are also known as compound lipids. ${ }^{9}$ The emphasis here will be on the phospholipids which have received wider acceptance in the area of drug delivery. A combination of these lipids tend to complement each other thereby giving the best drug delivery property and can be tailored towards controlled release formulation. Researchers in the field of drug delivery have used this combination for effective delivery of certain drug molecules. ${ }^{15,16}$

The study was aimed at evaluating ibuprofen loaded liposphere formulated with beeswax and phospholipid (Phospholipon ${ }^{\circledR}$ 90H) both in vitro and in vivo in order to improve the oral bioavailability of ibuprofen and reduce its ulcerative properties.

\section{MATERIALS AND METHODS}

Phospholipon $^{\circledR} \quad 90 \mathrm{H} \quad$ (P90H) $\quad$ (Phospholipids $\mathrm{GmbH}$, Germany) is a purified, deoiled, and granulated soy lecithin with phosphatidylcholine content of at least 90\%. Beeswax (Carl Roth, Germany), poloxamer-188 (P-188) (BASF, Germany), monobasic potassium phosphate, sodium hydroxide and concentrated hydrochloric acid (BDH, Poole, England), ibuprofen (a gift from Juhel Nig. Ltd. Enugu, Nigeria) and distilled water (Lion water, University of Nigeria Nsukka) were used as procured. Other reagents were of analytical grade and were used without further purification.

Formulation of the lipid matrix. The lipid matrix consisting of Phospholipon ${ }^{\circledR} 90 \mathrm{H}$ and beeswax in the ratio of 1:3 was prepared by fusion. ${ }^{3}$ Briefly, $25 \mathrm{~g}$ and $75 \mathrm{~g}$ of the various lipids were weighed and melted together at $10{ }^{\circ} \mathrm{C}$ above the melting point of the solid lipids to prevent the lipid memory effect; on a thermo-regulated water bath shaker (Heto, Denmark) and stirred until solidification to get lipid matrix.

Formulation of lipospheres. Lipospheres containing $5 \% \mathrm{w} / \mathrm{w}$ of the lipid matrix, $2 \% \mathrm{w} / \mathrm{w}$ of poloxamer 188 (P-188), 2\% w/w of polyvinyl alcohol (PVA) and enough distilled water to make $100 \% \mathrm{w} / \mathrm{w}$ dispersion were prepared by hot homogenization technique. ${ }^{3}$ Briefly, the aqueous phase containing P188 and PVA at same temperature was added to the molten lipid with gentle stirring using magnetic stirrer (SR 1 UM 52188, Remi Equip., India). The mixture was further dispersed with a homogenizer (Ultra-Turrax $^{\circledR}$ T18 basic digital; IKA, Staufen, Germany) at $12000 \mathrm{rpm}$ for $5 \mathrm{~min}$ to produce the hot primary emulsion. The liposphere suspension obtained after cooling at room temperature was filtered (Millipore filter, Delhi, India) to remove water. By adding increasing concentrations of IBU $(100,200$, and $400 \mathrm{mg})$ to the $5 \% \mathrm{w} / \mathrm{w}$ lipid matrix and following the above described procedure, IBUloaded lipospheres (batches A1-A3 respectively) were obtained. A batch of lipospheres containing no drug (unloaded lipospheres, which served as negative control) was similarly prepared and coded A-0. 
Characterisation of IBU-loaded lipospheres. The particle size of the lipospheres was determined by computerized image analysis of at least 100 lipospheres on a photomicroscope (Lieca, Germany). Each batch was mounted on a slide and observed under a light microscope. With the aid of the camera software, the perimeter of the particles corresponding to the particle sizes of the lipospheres were determined and average calculated. The particle morphologies were also observed and photographs taken. All determination was done one week post formulation.

Encapsulation efficiency \%. The EE (\%) of each batch was determined after $48 \mathrm{~h}$ of preparation. A $100 \mathrm{mg}$ quantity of each batch of the lipospheres were dispersed in a $100 \mathrm{ml}$ volumetric flask and allowed to equilibrate for $48 \mathrm{~h}$ at room temperature. The resultant mixture was shaken and filtered using $0.20 \mu \mathrm{m}$ pore size (Millipore ${ }^{\circledR}$ filter, Delhi, India). The filtrate was adequately analyzed for IBU content spectrophotometrically (Jenway, model 6504, UK) at $223 \mathrm{~nm}$. The amount of drug encapsulated in the lipospheres was calculated with reference to a standard Beer's plot for ibuprofen. The percentage EE was thereafter obtained using the formula:

$$
E E=\frac{A D L}{T D L} \times 100
$$

Where, ADL is the actual drug loading and TDL is the theoretical drug loading.

Loading capacity (LC). LC expresses the ratio between the entrapped drug and the total weight of the lipids. It was determined using Eq. 2:

$$
L C=\frac{Q_{D}-Q_{S}}{q_{I}} \times 100 \quad \text { Eq. } 2
$$

Where, $\mathrm{q}_{1}$ is the weight of lipid added in the formulation, $Q_{d}$ is the weight of ibuprofen added to the formulation and $Q_{s}$ is the amount of drug determined in supernatant after separation of the lipid and aqueous phase.

In vitro release study. The in vitro drug release profile of IBU-loaded lipospheres and conventional marketed formulation were studied using a dialysis membrane. Formulations were added to the dialysis membrane (MWCO $12 \mathrm{KDa}$, Himedia, India) and placed in a beaker containing $250 \mathrm{ml}$ of phosphate buffered saline (PBS, pH 7.4). The beaker was placed over a magnetic stirrer and the temperature of the assembly was maintained at $37 \pm 1{ }^{\circ} \mathrm{C}$ throughout the study. At $0.5,1,2,4,6,8,10,12,14,18$ and $20 \mathrm{~h}$ approximately, $5 \mathrm{ml}$ samples were withdrawn from the medium and replaced with an equal amount of freshly prepared PBS to maintain sink condition throughout the study. Samples were filtered using a $0.2 \mu \mathrm{m}$, (Millipore ${ }^{\circledR}$ filter, Delhi, India) and analyzed for IBU content using a UV-VIS spectrophotometer (Jenway, model 6405, UK) at $223 \mathrm{~nm}$.

\section{In vivo studies}

Animals. Rats weighing between 190 and $210 \mathrm{~g}$ were obtained from the animal house of Department of Biochemistry, University of Nigeria Nsukka, Nigeria. The rats were all kept on standard and conditioned animal cages and left for one week to acclimatize to the new laboratory conditions. They were fed with standard rations. The animal experiments in this study complied with the regulations of the Committee on Ethics on the use of Laboratory Animals of the University of Nigeria and were in compliance with the Federation of European Laboratory Animal Science Association and the European Community Council Directive of November 24, 1986.

Anti-inflammatory studies. The anti-inflammatory activity of the IBU-loaded lipospheres was evaluated using the rat paw edema model. Rats were divided into four groups of five animals each. Rats in group I served as control and received normal saline (NS) $5 \mathrm{ml} / \mathrm{kg}$; group II received pure sample of IBU $(50 \mathrm{mg} / \mathrm{kg})$ and served as the reference group while group III received marketed sample $50 \mathrm{mg} / \mathrm{kg}$ for the purpose of comparison. Group IV was treated with IBU-loaded liposphere $(50 \quad \mathrm{mg} / \mathrm{kg})$. All administrations were done orally. Thirty minutes post-treatment, oedema was induced by injection of $0.1 \mathrm{ml}$ of $0.2 \%$ of $\gamma$-carrageenan into the sub plantar region of the right hind paw of the rats. The volume of distilled water displaced by treated right hind paw 
of the rats were measured using a digital plethysmometer (Ugo Basil, USA) before $\gamma$-carrageenan administration and at $0.5,1,2,4$ and 8 $\mathrm{h}$ post $\gamma$-carrageenan injection, using the plethysmometer. The amount of paw swelling was determined and expressed as percent inhibition using the following equation:

$\%$ Inhibition $=\frac{I_{C}-I_{t}}{I_{C}} \times 100$

Where, $I_{c}=$ is the edema rate of control group, $I_{t}=$ is the edema rate of the treated group.

Acute analgesic activity of the formulation. Analgesic activity was tested in rats using the method described by earlier researcher ${ }^{17}$ based on tail flick method using analgesiometer (Techno, India). Briefly, twenty five rats of either sex of average weight $210 \pm 0.11 \mathrm{~g}$ were grouped into five groups of five animals per group. The optimized formulation (A-3) was used for this study. Our selection was based on the in vitro preliminary evaluation and \% EE. Rat in group I were treated with marketed sample (MKT-S) of IBU (50 mg/kg), group II, III and IV were treated with three different doses of batch A-3 $(10,20$ and $50 \mathrm{mg} / \mathrm{kg}$, respectively) and group $\mathrm{V}$ was treated with pure sample (PS) $(50 \mathrm{mg} / \mathrm{kg})$ and served as reference group. Thirty minutes after the last dose administration pain threshold values of the animals were evaluated. Briefly, the terminal (junction between proximal $2 / 3$ and distal $1 / 3$ ) portion of the tail was kept at $0.5 \mathrm{~cm}$ above the heated tantalums wire, and the time taken to flick and remove the tail was noted. To validate the accuracy of this method and timing, we carried out this study under an observer who was unaware of the treatment groups. The "pain threshold" was defined as the time when the animal first showed evidence of the sensation of pain, indicated by shaking of tail and/or the initial signs of paw withdrawal and/or vocalization. The tail was not kept for more than 40 seconds to avoid burn injury.

Pharmacokinetics studies. Fasted albino rats of both sex and average weight, $210 \mathrm{~g} \pm 0.12$ were divided into three groups of five rats per group. Rats in group I were intragastrically administered $2.0 \mathrm{ml}$ of $20 \mathrm{mg} / \mathrm{kg}$ of the IBU formulation in double distilled water; group II rats received marketed IBU formulation in a dose of $20 \mathrm{mg} / \mathrm{kg}$ body weight and group III rats received pure sample of IBU $(20 \mathrm{mg} / \mathrm{kg}$ body weight) which serve as positive control. At predetermined time intervals of $0,0.5,1,2,4,6$ and 8 $\mathrm{h}$, blood samples were collected from the orbital sinus of the eye to an empty EDTA container under mild ether anesthesia. The blood samples were centrifuged at $50 \mathrm{rpm}$ to extract the serum. The extracted samples were further subjected to lowspeed centrifugation, and the supernatant collected and stored for further analysis.

Pharmacokinetic analysis was performed using a bioavailability Calc 2002 pharmacokinetic analysis computer program (Korea Food \& Drug Administration, Korea). Area under the curve (AUC) was calculated using the linear trapezoidal rule. Maximum plasma concentration $\left(\mathrm{C}_{\max }\right)$ and the time needed to reach the maximum plasma concentration $\left(\mathrm{T}_{\max }\right)$ were determined directly from the concentration-time data. The AUC was used as an index to measure the bioavailability of prepared ibuprofen tablets and the reference drug as discussed earlier. ${ }^{18}$ All experimental data points were expressed as the mean ( $\pm \mathrm{SD}$ ). The corresponding mean pharmacokinetic parameters $( \pm \mathrm{SD})$ were calculated from individual estimates in the animal group. Significant differences between the experiments were calculated using a student's t test (unpaired) (Sigma plot $^{\circledR}$, version 11, Rock Ware Inc, USA), using a twotailed distribution and a two-sample unequal variance (heteroscedastic) method. A value of $p<0.05$ was considered statistically significant.

Data analysis. Data were expressed as mean values \pm standard deviation. The student's $t$-test was applied to study the significance of the difference between two groups (One way Analysis of Variance (ANOVA), $p$ value of less than 0.05 was considered to be statistically significant.

\section{RESULTS}

An optimized combination of two different lipids with different structural characteristics was employed 
in the preparation of lipospheres based on matrix system using a slightly modified multiple emulsion system.

Morphological evaluation. The shapes of the particles were smooth and spherical (Figures $1 \mathrm{~A}-0$ to
A-3). There was a slight change in the shape as the concentration of the drug increased an indication that the drug loading affected the shape as well as the size of the particles.
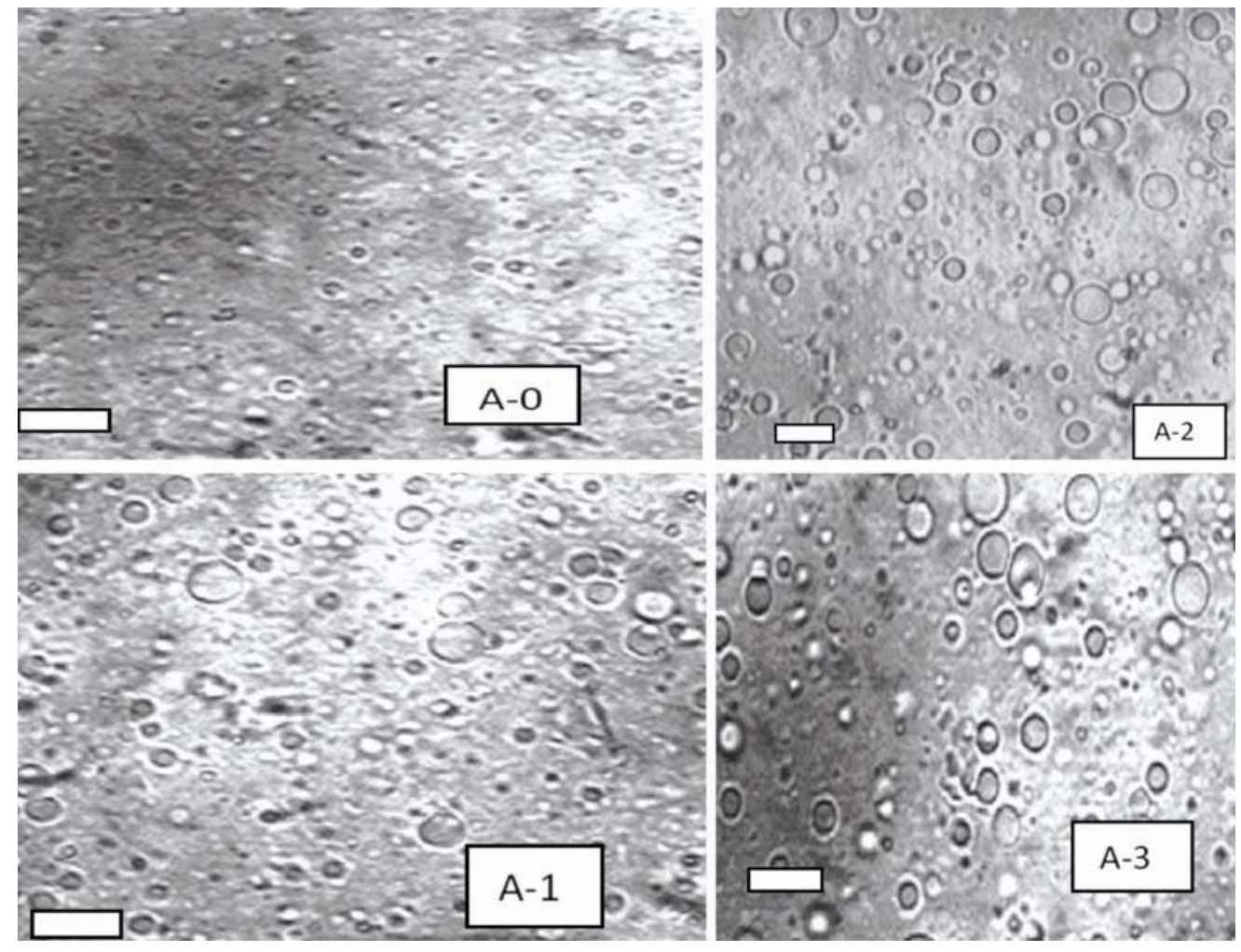

Figure 1. Photomicrographs of representative batches of lipospheres containing $5 \% \mathrm{w} / \mathrm{w}$ lipid matrix: A- 0, A-1, A-2 and A-3 contain 0,100 , 200 and $400 \mathrm{mg}$ of IBU, respectively. Bar represents $70 \mu \mathrm{m}$.

Particle size analysis. The particle sizes of the lipospheres are shown in table 1 . The particle sizes were in the range of $137 \pm 0.1$ to $178 \pm 0.3 \mu \mathrm{m}$, with batch A-3 having the largest size. The unloaded batch A-0 showed the smallest particle size $(96.3 \pm 0.10$ $\mu \mathrm{m})$.

Encapsulation efficiency (\%). The IBU encapsulation efficiency (\%) was observed to be dependent on the amount of drug incorporated into the lipospheres. The drug entrapment efficiencies of all batches of the lipospheres were in the range of 89.4 to $97.9 \%$, with lipospheres formulated with higher amount of ibuprofen $(400 \mathrm{mg}$ ) recording the highest drug entrapment. The general pattern was that
IBU encapsulation increased with increase in the concentration of IBU.

Table 1. Some physicochemical properties of IBU-loaded with liposphere.

\begin{tabular}{llll}
\hline $\begin{array}{l}\text { Formulation } \\
\text { code }\end{array}$ & PS $(\mu \mathrm{m})$ & $\% \mathrm{EE}$ & LC \\
\hline A-0 & $96.3 \pm 0.10$ & $* * *$ & $* * *$ \\
A-1 & $101 \pm 0.30$ & $89.4 \pm 0.21$ & $33.3 \pm 0.1$ \\
A-2 & $126 \pm 0.10$ & $94.9 \pm 0.34$ & $47.1 \pm 0.4$ \\
A-3 & $178 \pm 0.30$ & $97.9 \pm 0.30$ & $46.6 \pm 0.0$ \\
\hline
\end{tabular}

$\mathrm{PS}=$ Particles size, $\mathrm{EE}=$ encapsulation efficiency and $\mathrm{LC}=$ Loading capacity. Note: A1- A3 were the formulation loaded with 100, 200 and $400 \mathrm{mg}$ of IBU respectively. A-0 is the unloaded batch of liposphere (without IBU) and $* * *$ parameters not evaluated. 
In vitro release of IBU from the lipospheres. The in vitro release profiles of IBU in PBS (pH 7.4) was high, indicating significant release of IBU from all batches of the formulation as shown in figure 2 . Batch A3 formulation gave the maximum release of $96.9 \%$, while A1 gave the least (75\%) and batch A2 released $81 \%$ of IBU at $20 \mathrm{~h}$.

Anti-inflammatory activity. The antiinflammatory results are depicted in figure 3. Results indicate that anti-inflammatory activity of IBUloaded lipospheres was comparable to that of pure sample (PS) at same concentration. The activity of IBU-loaded lipospheres at a dose of $50 \mathrm{mg} / \mathrm{kg}$ was approximately 1.5-times higher than that of the marketed sample (MKT-S) and twice that of PS. It is noteworthy that the activity of the formulated lipospheres was concentration dependent as presented in figure 3.

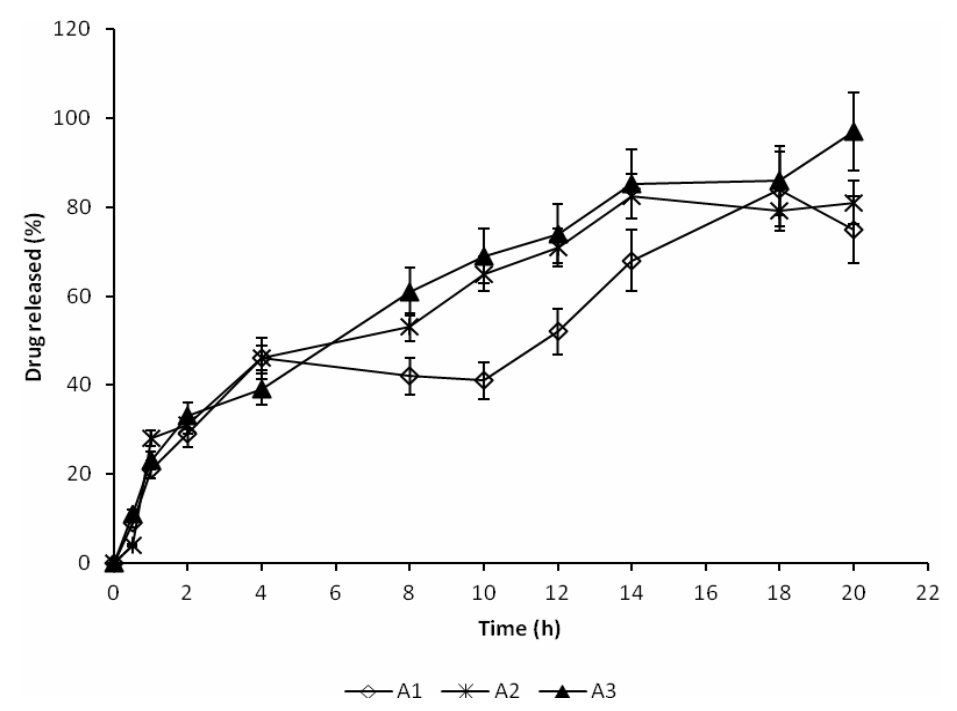

Figure 2. Release profile of IBU from the lipospheres in phosphate buffered saline (PBS, pH 7.4).

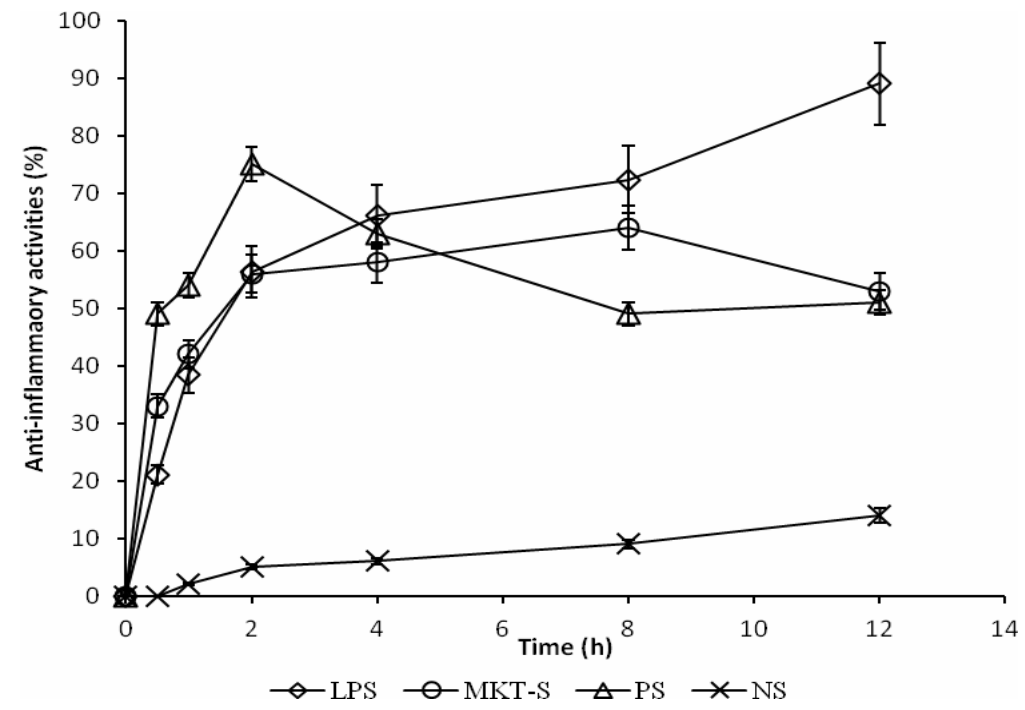

Figure 3. Anti-inflammatory activities of IBU-loaded lipospheres in a rat model. Each data point represents the mean $\pm \operatorname{SEM}(n=5)$. 
Analgesic study. Figure 4, showing the plot of pain threshold values of the inflamed hind paw to the analgesic activities demonstrated that lipospheres heat compared to MKT-S treated group and PS loaded with IBU significantly $(p<0.05)$ increased the treated group, at all doses tested.

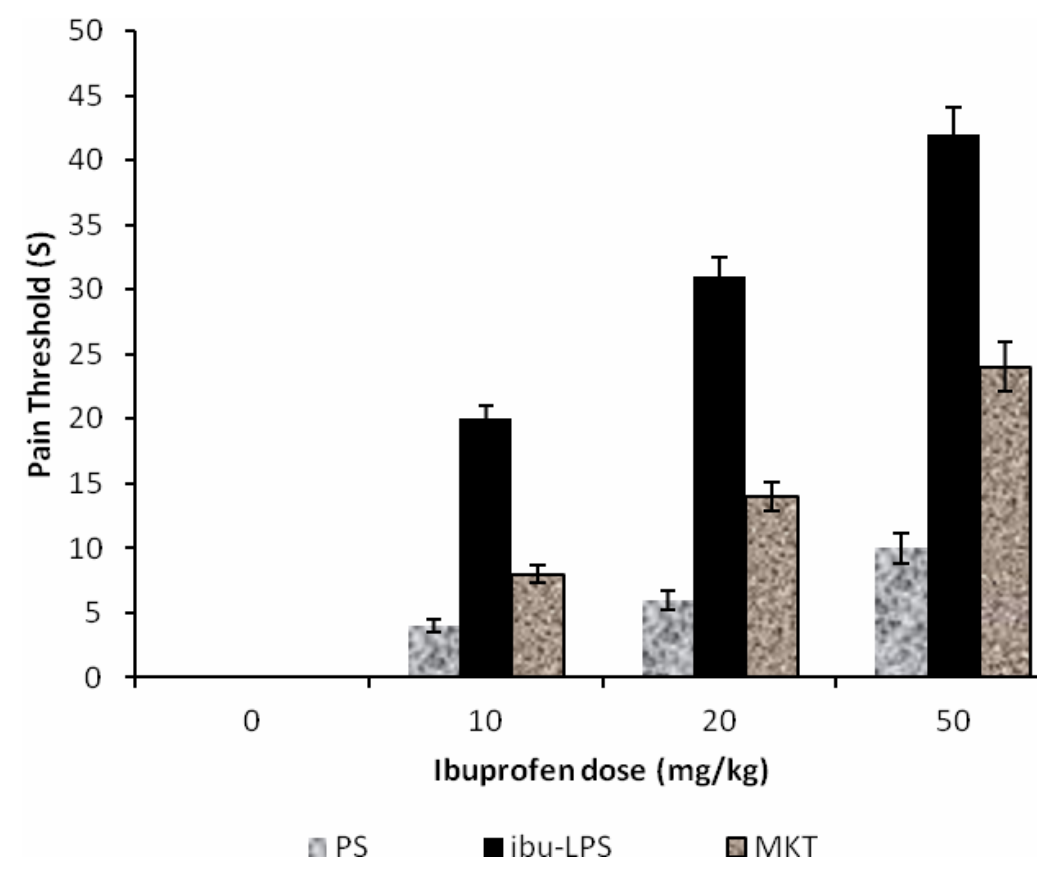

Figure 4. Comparison of acute analgesic activity of IBU-loaded lipospheres, PS and MKT-S in rat model. Each data point represents the mean $\pm \operatorname{SEM}(n=5)$.

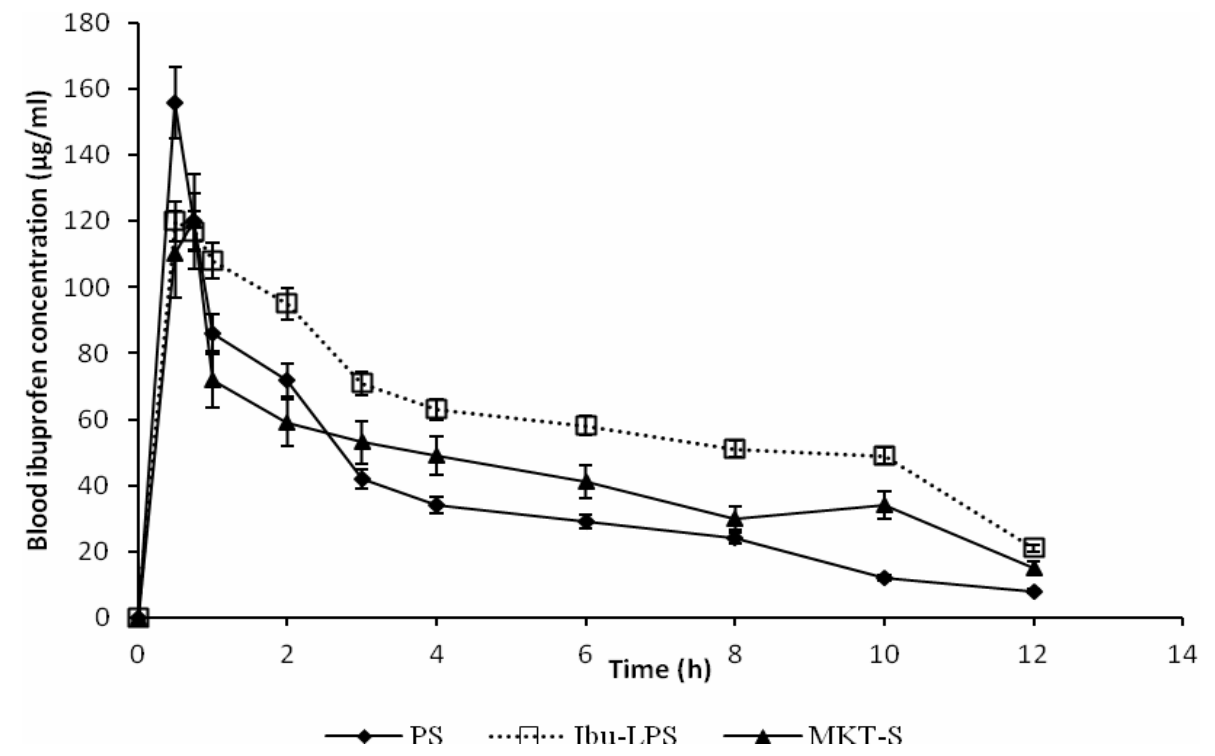

Figure 5. Changes of IBU concentration in blood over 12-h study period of rats orally administered with IBU-loaded lipospheres, ibuprofen pure sample (PS) and ibuprofen marketed sample (MKT-S) at a dose of $20 \mathrm{mg} / \mathrm{kg}$. All data point represents the mean $\pm \operatorname{SEM}(\mathrm{n}=5)$. 
Pharmacokinetic study. Results of the $12 \mathrm{~h}$ evaluation of IBU blood concentrations were depicted in figure 5 . The result indicates that the concentration of IBU in the blood increased rapidly and reached peak values within the first few minutes in all tested formulations. There was a sharp fall in the PS as compared to IBU-loaded lipospheres and MKT-S. On the contrary, lipospheres loaded with IBU showed a slow decrease in plasma concentration when compared to MKT-S, an indication that the carrier inhibited the clearance of IBU from systemic circulation. The $\mathrm{C}_{\max }$ for PS was $158 \mu \mathrm{g} / \mathrm{ml}$ at $30 \mathrm{~min}$ while IBU-loaded lipospheres formulation was 124 $\mu \mathrm{g} / \mathrm{ml}$ at $15 \mathrm{~min}$ and that of MKT-S was $120 \mu \mathrm{g} / \mathrm{ml}$ at $30 \mathrm{~min}$. The high peak observed in PS was not sustained compared to IBU-loaded lipospheres and MKT-S. The AUCs were 134, 678 and $1897(\mu \mathrm{g}$ $\mathrm{h} / \mathrm{ml}$ ) and the $\mathrm{T}_{1 / 2}$ were $2.4 \pm 0.66 \mathrm{~h}, 4.2 \pm 3.12 \mathrm{~h}$ and $8.5 \pm 1.20 \mathrm{~h}$ for PS, MKT-S and IBU-loaded lipospheres, respectively.

\section{DISCUSSION}

The main aim of lipospheres delivery system is to deliver the drug to the target tissues intact and enhance bioavailability. Thus, the ability of the lipospheres to accommodate active molecules is an important property that determines the efficacy of the delivery method. It can be expressed by the entrapment efficiency (EE \%) and loading capacity (LC). The EE (\%) is simply explained as the ratio between the weight of entrapped drug and the total weight of drug added to the carrier system, while LC expresses the ratio between the entrapped drug by the lipid and the total weight of the lipids. ${ }^{17,18}$ Table 2 shows the EE\% and the LC obtained for the various batches of the lipospheres after 1 month of storage. Parameters such as solubility of the drug, surfactant type and the production method were the rate determining step for the EE \% and LC. The EE (\%) of the formulation were $89.4 \pm 0.21,94.9 \pm 0.34$ and $97.9 \pm 0.30$ for $\mathrm{A} 1$ to $\mathrm{A} 3$, respectively, while that of LC were $33.3 \pm 0.1,47.1 \pm 0.4$ and $46.6 \pm 0.0$, respectively (Table 1). The values obtained for the EE (\%) was very high and could be attributed to the structural distortion of the lipid matrix which helped to incorporate more drug within the matrix in addition to surface modification. ${ }^{19,20}$ The phospholipid bilayer structure formed around the lipid core may increase the drug loading capacity, as biologically important molecules (drug) can be anchored on the colloidal particle surface. Surface modification also enables stabilization of colloidal particles especially the microparticles formed in aqueous medium. ${ }^{21,22}$ However, there are slight variation in $\mathrm{EE} \%$ and $\mathrm{LC}$, which may be attributed to the varied concentration of drug used in the formulation. All the same, higher $\mathrm{EE} \%$ values were obtained for all the batches of the formulations. There was insignificant difference $(p<0.05)$ in the $\mathrm{EE} \%$ obtained for all the batches (Table 1).

The release profiles shown in figure 2 indicated that the formulations showed a controlled release pattern with batch A-3 gave the maximum release of $96.9 \%$ in $20 \mathrm{~h}$, while batches $\mathrm{A}-1$ and $\mathrm{A}-2$ gave maximum release of 75 and $81 \%$ in $20 \mathrm{~h}$ respectively. There was a correspond increase in the amount of drug release when the drug concentration in the formulation was increased. The formulations showed an initial rapid drug release which may be attributed to the formulation method or the carrier. Generally, drug release from a lipid matrix, solidified reverse micellar solution or liposphere follows four sequential steps: water penetrating into the lipid carrier, dissolution of the drug, migration of the drug from the core matrix and erosion of the lipid. However, for poorly water-soluble drugs such as ibuprofen, there is always a delay in the release of poorly water soluble drug, coupled with poor water penetratability into the lipid core of the microparticles, thus dissolution of the drug is a key rate-limiting step in the release process. The drug migration in this kind of formulation may be due to combination of erosion and diffusion to the outer surface of the lipid, hence release into the medium. ${ }^{23}$

The results of anti-inflammatory and analgesic properties of the IBU-loaded lipospheres presented in figures 3 and 4 showed the ability of the formulations to reverse inflammation and decrease pain sensitivity 
associated with external pressure applied to the rats. This effect was consistently enhanced in all the formulations containing IBU compared to marketed sample. Indeed, in contrast to the tested batches of the formulations, marketed sample of IBU failed to appreciably reverse the inflammation induced on the rats. However, there was a significant difference between the effects produced by the marketed sample and by the pure drug. Generally, the IBU-loaded lipospheres showed higher inhibition of oedema than the reference (plain ibuprofen). This was due to increased absorption of drug in vivo in the presence of the lipidic carrier. Drugs suspended in lipid matrix have been shown, in most cases, to be better absorbed than those incorporated in conventional solid dosage forms due to the ease of wetting of hydrophobic drug particles in the presence of lipid matrix. ${ }^{24}$ The presence of surfactant in the formulation also promoted drug release. ${ }^{25}$ The same trend was observed in the analgesic evaluation. It was further observed that the formulated ibuprofen had a higher threshold value for pain than the control. The results indicated that the released drug was effectively absorbed into the biological system thereby blocking the pain receptor sites which ultimately improved resistance to pain. However, the effect was concentration-dependent.

The absorption of a given drug depends on the balance of its solubility, hydrophobicity, surface area release of drug in the aqueous environment of the gastrointestinal lumen and its capability to diffuse across the lipophilic apical membrane of enterocytes. Generally, drugs have to be dissolved in order to attain sufficient bioavailability. The solubility of a given drug directly depends on its solid-state properties, e.g. particle size, crystalline or amorphous state, wettability, and others. ${ }^{24}$

A primary requirement of a lipid-based formulation is its ability to retain a poorly soluble substance in a solubilized state and to enhance solutesolvent interactions also after mixing with endogenous solubilizers, such as bile acids or phospholipids produced naturally in the body or after intra-luminal processing prior to absorption. In this study, we compared the bioavailability of ibuprofenloaded lipospheres formulations to that of an equivalent dose of pure IBU sample and marketed sample.

A pharmacokinetic analysis of the appearance of IBU in the blood was performed within 720 min after fasted rats were orally administered with lipospheresloaded with IBU at a dose of $100 \mathrm{mg} / \mathrm{kg}$. The higher dose of drug-loaded lipospheres was used in this pharmacokinetic study to ensure good bioavailability detection in the plasma. Pharmacokinetic analysis revealed that $\mathrm{P} 90 \mathrm{H}$ association altered both the uptake and decay of ibuprofen in the blood. The IBU formulation containing the lipid matrix resulted in a decrease in the initial peak IBU blood levels attained 15 min after oral administration of the formulation. However, in contrast, the rapid decrease in the pure sample of IBU after attaining initial peak, the clearance rate became very slow, while that of the formulated ibuprofen showed much slower decrease from the peak with sustained high plasma concentration over $10 \mathrm{~h}$ period. This was due to a 2 to 3 -fold increase in the circulating $T_{1 / 2}$ of formulated ibuprofen. There was a significant $(p<0.05)$ differences in the plasma clearance rate between the formulated IBU and pure sample. It was noted that the AUC for IBU-loaded lipospheres $(1897 \mu \mathrm{g} \mathrm{h} / \mathrm{ml})$ was higher compared to PS $(134 \mu \mathrm{g} \mathrm{h} / \mathrm{ml})$ and the MKT-sample $(678 \mu \mathrm{g} \mathrm{h} / \mathrm{ml})$. All these were direct impact of the lipid carrier used in the formulation. However, it is most likely that the phospholipid acted as a depot that delayed release of IBU from the gastrointestinal (GI) lumen into the blood or altered the clearance of IBU. The results here are in agreement with previous studies where addition of phospholipd to NSAIDs improved the therapeutic effect. $^{26}$

\section{CONCLUSIONS}

The design and preparation of lipospheres based on lipid blends is relatively new and has gained wider acceptance in the field of pharmaceutical drug formulators that seeks to exploit the attractive properties of lipid carriers to improve the delivery of 
therapeutic molecules. This study has demonstrated that beeswax could be very useful in the preparation of stable lipospheres when combined with phospholipids. Overall, the formulations showed good physicochemical properties. The lipospheres exhibited good anti-inflammatory and analgesic properties as well as minimal ulcerogenic potentials. The plasma clearance of the IBU-loaded liposphere was significantly $(\mathrm{p}<0.005)$ higher than the controls. Thus, lipospheres delivery system could be further explored for the delivery of ibuprofen.

\section{ACKNOWLEDGEMENTS}

We thank Phospholipid GmbH, Koln, Germany and BASF AG, Ludwigshafen, Germany for providing samples of Phospholipon ${ }^{\circledR}$ 90H. Dr. MA Momoh wish to thank Pharm. Abdulmumin Z Abubakar of Department of Pharmacognosy and Drug Development, Ahmadu Bello University Zaria for his immense contribution in shaping this work.

\section{Declaration of interest}

The authors state no conflicts of interest and have received no funding for the research or in the preparation of this manuscript.

\section{REFERENCES}

1. Sachan, K.N., Bhattacharya, A., Purshka, S. and Mishra, A. 2009. Biopharmaceutics classification system: A strategic tool for oral drug delivery technology. Asia. J. Pharm. 8, 7681.

2. Abdel-Mottaleb, M.A., Neumann, D. and Lamprecht, A. 2010. In vitro drug release mechanism from lipid nanocapsules (LNC). Int. J. Pharm. 390, 208-213.

3. Momoh, M.A., Kenechukwu, F.C. and Attama, A.A. 2013. Formulation and evaluation of novel solid lipid microparticles as a sustained release system for the delivery of metformin hydrochloride. Drug Deliv. Early Online: (2013), 1-10

4. Harms, M. and Müller-Goymann, C.C. 2011. Solid lipid nanoparticles for drug delivery. J. Drug. Del. Sci. Tech. 21, 89-99.

5. Satheesh, B.N., Prabakaran, L., Sundareswara, K.C., Gayathri, R. and Saravanan, T. 2011. Formulation design and in vitro evaluation of azithromycin loaded lipospheres using melt dispersion technique. J. Pharm. Res. 4, 4069-4071
6. Domb, A.J. 1995. Long acting injectable oxytetracyclinelipospheres formulation. Int. J. Pharm. 124, 271-278.

7. Del Pozo-Rodriguez, A., Delgado, D., Solinís, M.A. and Gascón, A.G. 2011. Lipid nanoparticles as vehicles for macromolecules: nucleic acids and peptides. Drug. Deliv. 5, 214-226.

8. Almeida, A.J. and Souto, E.B. 2007. Solid lipid nanoparticles as a drug delivery system for peptides and proteins. Adv. Drug Deliv. Rev. 59, 478-490.

9. Puri, D., Bhandari, A., Sharma, P. and Choudhary, D. 2010. Lipid nanoparticles (SLN, NLC): A novel approach for cosmetic and dermal drug delivery. Pharm. J. Global. Pharma. Tech. 2, 1-15.

10. Wang, M.T., Jin, Y., Yang, Y.X., Zhao, C.Y., Yang, H.Y., $\mathrm{Xu}$, X.F., Qin, X., Zhang, Z.R. and Huang, Y. 2010. In vivo biodistribution, anti-inflammatory, and hepatoprotective effects of liver targeting dexamethasone acetate loaded nanostructured lipid carrier system. Int. J. Nanomed, 5, 487497.

11. Moneghini, M., Bellich, B. and Baxa, P. 2008. Microwave generated solid dispersions containing ibuprofen. Int. J. Pharm. 29, 361:125.

12. Pang, J., Luan, Y. and Li, F. 2010. Ionic liquid-assisted synthesis of silica particles and their application in drug release. Mater Lett. 64, 2509-2512.

13. Newa, M., Bhandari, K.H. and Li, D.X. 2007. Preparation, characterization and in vivo evaluation of ibuprofen binary solid dispersions with poloxamer 188. Int. J. Pharm. 7, 343: 228-237.

14. Jain, J.A. 2000. The manufacturing techniques of various drug loaded biodegradable poly (lactide-co-glycolide) (PLGA) devices. Biomat. 21, 2475-2490.

15. Battaglia, L. and Gallarate, M. 2012. Lipid nanoparticles: State of the art, new preparation methods and challenges in drug delivery. Expert. Opin. Drug. Deliv. 9, 497-508.

16. Tang, Y. and Singh, J. 2008. Controlled delivery of aspirin: Effect of aspirin on polymer degradation an in vitro release from PLGA based phase sensitive systems. Int. J. Pharm. 357, 119-125.

17. Nnamani, P.O., Attama, A.A., Ibezim, E.C. and Adikwu, M.U. 2010. SRMS142-based solid lipid microparticles: Application in oral delivery of glibenclamide to diabetic rats. Eur. J. Pharm. Biopharm. 76, 68-74

18. Attama, A.A. and Muller-Goymann, C.C. 2007. Investigation of surface modified solid lipid nano-container formulated with a heterolipid templated homolipid. Int. J. Pharm. 334, 179-189.

19. Ye, J.S., Wang, Q., Zhou, X.F. and Zhang, N. 2008. Injectable actarit-loaded solid lipid nanoparticles as passive targeting therapeutic agents for rheumatoid arthritis. Int. J. Pharm. 352, 273-279.

20. Schwarz, C. and Mehnert, W. 1999. Solid lipid nanoparticles (SLN) for controlled drug delivery: Drug incorporation and physicochemical characterization. J. Microencapsul 16, 205. 
21. Doktorovova, S. and Souto, B.S. 2009. Nanostructured lipid carrier-based hydrogel formulations for drug delivery: a comprehensive review. Exp. Opin. Drug. Deliv. 6, 165-76.

22. Yang. S.C., Lu, L.F., Cai, Y., Zhu, J.B., Liang, B.W. and Yang, C.Y. 1999. Body distribution in mice of intravenously injected camptothecin solid lipid nanoparticles and targeting effect on brain. J. Cont. Rel. 59, 299-307.

23. Liu, Y., Pan, J. and Feng, S.S. 2010. Nanoparticles of lipid monolayer shell and biodegradable polymer core for controlled release of paclitaxel: Effects of surfactants on particles size, characteristics and in vitro performance. Int. J. Pharm. 395, 243-250.
24. Attama, A.A., Okafor, C.O., Builders, P.F. and Okorie, O. 2009. Formulation and in vitro evaluation of a PEGylated microscopic lipospheres delivery system for ceftriaxone sodium. Drug Deliv. 16, 448-616.

25. Attama, A.A. and Nkemnele, M.O. 2005. In vitro evaluation of drug release from self micro-emulsifying drug delivery systems using a biodegradable homolipid from Capra hircus. Int. J. Pharm. 304, 4-10.

26. Dunjic, B.S., Axelson, J., Ar'Rajab, A., Larsson, K. and Bengmark, S. 1993. Gastroprotective capability of exogenous phosphatidylcholine in experimentally induced chronic gastric ulcer in rats. Scand J. Gastroenterol 28, 89-94. 